\title{
Sensitivity Analysis of Torque Ripple Reduction of Synchronous Reluctance and Interior PM Motors
}

\author{
Nicola Bianchi $^{(x)}$, Michele Degano $^{(o)}$ and Emanuele Fornasiero ${ }^{(x)}$ \\ (x) Dept. of Industrial Engineering, Univ. of Padova, via Gradenigo 6/A, 35131 PD (ITALY) \\ ${ }^{(o)}$ Dept. of Electrical, Electronic and Computer Engineering, Univ. of Trieste, 34127 TS (ITALY)
}

\begin{abstract}
The main drawback of reluctance machines is a high torque ripple, due to the interaction between the stator magneto-motive force and the rotor structure. Adopting a rotor configuration characterized by several flux barriers per pole, there is a high influence of the rotor geometry on the machine performance, in terms of both average torque and ripple. An optimization is often required to determine the optimal rotor geometry so as to achieve a high and smooth torque. Then, the geometry determined above should guarantee good performance for various operating points (i.e., changing the current amplitude and phase), as well as for small variations of the geometry. This paper investigates this aspect, showing the results of optimizations carried out on various machines. The impact of the geometry parameters is taken into account and the sensitivity of the optimal solution to the geometry variation is pointed out. The paper highlights the difficulty to get a robust geometry as far as the torque ripple reduction is concerned. Finally, a few experimental results on a Synchronous Reluctance motor prototype will be presented, compared with Finite Element Analysis simulations for validation.
\end{abstract}

\section{INTRODUCTION}

In order to exhibit a proper torque, the synchronous reluctance machine (SynREL) is characterized by a small airgap and a high anisotropic rotor. Several rotor flux barriers force the flux lines to flow through given iron paths. For the purpose to saturate the iron bridges (both inner and outer) and to increase the power factor, permanent magnets (PM) are sometimes inset within the flux barriers. In this case, the machine is referred to as PM assisted reluctance machine (PMAREL), or interior permanent magnet (IPM) machine [1], [2]. Fig. 1 shows a four-pole SynREL rotor characterized by three flux barriers per pole.

Adopting a rotor configuration characterized by several flux barriers per pole, there is a high influence of the rotor geometry on the machine performance, in terms of both average torque and ripple. Therefore, an optimization is often required to the aim of determining a rotor geometry achieving a high and smooth torque. The optimal geometry should guarantee good performance for various operating points (i.e., changing the current amplitude and phase). In addition, small geometry variations, due to mechanical tolerance, wear of the machine tools, manufacturing or assembling inaccuracy, and so on, should only marginally affect the performance of the optimal machine. This paper investigates this aspect, showing the results of various optimizations carried out on different machines.

The impact of various geometry parameters is taken into

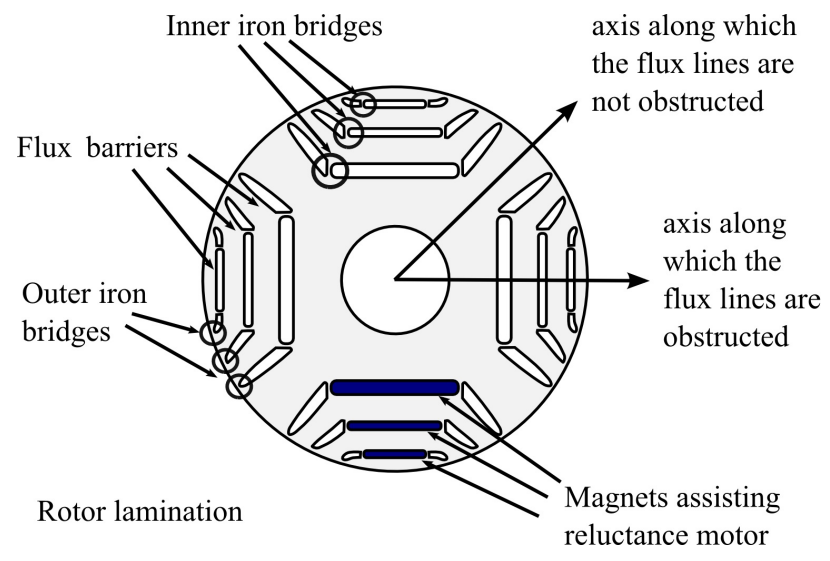

Fig. 1. Sketch of a four-pole synchronous reluctance rotor with three flux barriers per pole. The synchronous PM assisted reluctance motor is achieved when PMs are inset in the flux barriers.

account. The difficulty to get a robust geometry as far as the torque ripple reduction is highlighted.

\section{A BRIEF REVIEW OF TECHNIQUES TO REDUCE TORQUE RIPPLE OF SYNREL AND IPM MACHINES}

A common drawback of the SynREL and IPM machines is their high torque ripple [3]. This is caused by the interaction between the spatial harmonics of magneto-motive force (MMF) due to the stator currents and the rotor geometry. The main harmonic of stator MMF is synchronous with the rotor and produces the average electromagnetic torque. The other harmonics are not synchronous and cause variations of the flux across the flux barriers, that is, oscillations of the rotor magnetic potential. The main effect is a high torque ripple.

In [4] it has been shown that the rotor skewing (commonly adopted in PM machines [5], [6]) is not enough to smooth the torque. In any case, only a step-skewing is possible when PMs are used: the rotor is split in two or more parts, each of them is skewed with respect to the others. It has been also shown that a reduction of the torque ripple can be achieved by means of a suitable choice of the number of flux barriers with respect to the number of stator slots. In this case the flux barrier ends are uniformly distributed along the airgap (similarly to the stator slot distribution). In [7] and then in [8], the flux barriers are shifted from their symmetrical position. In this way, a sort of compensation of the torque harmonics is achieved. This technique is similar to that proposed in [9] for cogging torque 
reduction in surface-mounted PM motors.

Alternatively, a strategy to compensate the torque harmonics of the SynREL motor is presented in [10] by adopting two different flux barrier geometries in the same lamination, the resulting motor is referred to as "Machaon" motor (the name of a butterfly with two large and two small wings), since the flux barriers of the adjacent poles are large and small alternatively. A picture of a "Machaon" rotor lamination is shown in Fig. 2. In this case, not only the geometry of the flux-barriers is different in the adjacent poles, but also the number of the flux-barriers per pole. In the middle of each flux barrier, small PMs (the assisting PMs) can be added so as to saturate the iron bridges and to increase the power factor.

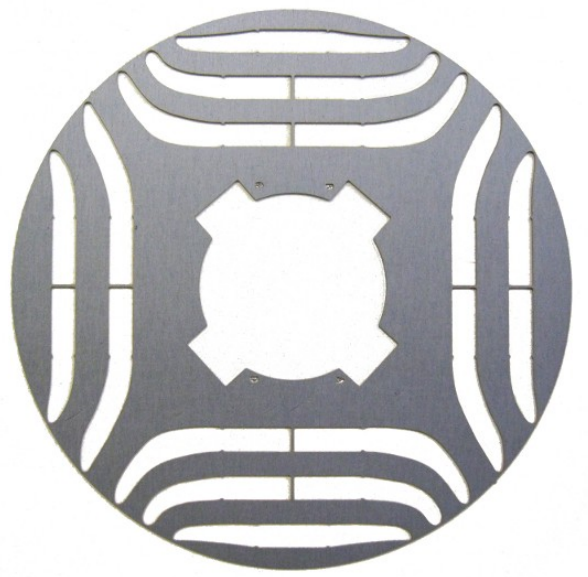

Fig. 2. Photo of a Machaon rotor lamination, characterized by the combination of two and three flux barriers per pole.

\section{A. Reference geometries}

Besides investigating the torque behavior, it is important to establish the influence of the various design parameters. Fig. 3 shows the main variables in the design space:

- $D$ is the inner diameter;

- $h_{s o}$ is the slot opening height;

- $w_{s o}$ is the slot opening width.

- $g$ is the airgap;

- $\vartheta_{b 1}, \vartheta_{b 2}$, and $\vartheta_{b 3}$ are the flux barrier angles;

- $L_{a i r}$ is the total thickness of the three flux barriers along the rotor $q$-axis; $L_{a i r}+L_{f e}$ represents the rotor radius.

Three different 36-slot 4-pole SynREL machines are considered in this study. Table I reports the main dimensions. Two of them $(A$ and $C$ ) have a symmetrical rotor, while the third one $(B)$ has a Machaon rotor structure. In order to guarantee a proper saliency ratio an Integral Slot Distributed Winding (ISDW) has been chosen. Since these machines present an high anisotropic rotor, has been demonstrated that this winding arrangement is the most effective solution comparing to Fractional Slot Concentrated winding (FSCW) [22], [23].

The saturation of the machine is strongly dependent by the thickness of the flux barriers. It is common to define an insulation coefficient $k_{a i r}$ as a ratio between $L_{a i r}$ and the iron thickness along the rotor $q$-axis (neglecting shaft radius). Each flux barrier's thickness (reported in Table I) has been determined through FE test simulations, in order to obtain a desired saturation level of the iron paths.

The thickness of the iron bridges of each rotor has been chosen according to the maximum speed required for each application. For the sake of comprehension, referring to Fig. 1, the inner iron bridges has mainly to guarantee a robust structure and resist to the centrifugal forces insisting on rotor parts. Also the outer iron bridges, in the following sections called iron ribs, even if they are less mechanically stressed with respect to the outer ones, have a structural function. Some details on the impact of the iron ribs thickness with respect to torque ripple are highlighted in Section III.

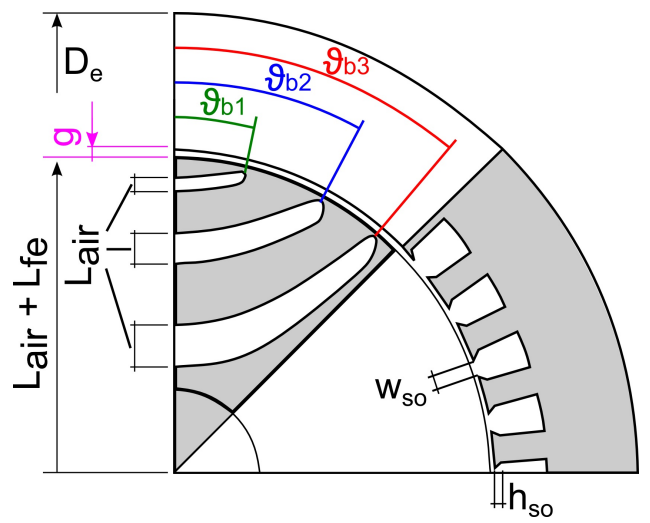

Fig. 3. Layout of the variable parameters.

TABLE I

MAIN GEOMETRICAL DIMENSIONS

\begin{tabular}{ccccc}
\hline Motor & $A$ & $B$ & $C$ & unit \\
\hline$D_{e}$ & 340 & 200 & 135 & $\mathrm{~mm}$ \\
$D_{r} e$ & 230 & 125 & 80 & $\mathrm{~mm}$ \\
$L_{\text {stk }}$ & 250 & 70 & 60 & $\mathrm{~mm}$ \\
Poles & 4 & 4 & 4 & - \\
Slots & 36 & 36 & 36 & - \\
$g$ & 0.7 & 0.5 & 0.3 & $\mathrm{~mm}$ \\
$k_{\text {air }}$ & 0.4 & $0.4 / 0.45$ & 0.4 & $\mathrm{~mm}$ \\
\hline$T_{n}$ & 260 & 20 & 7 & $\mathrm{Nm}$ \\
\hline
\end{tabular}

\section{B. Validation by means of experimental results}

Before comparing the torque behaviour of different solutions, the finite element predictions are compared with experimental results. Several tests have been carried out and the results are compared with finite element (FE) simulations. As an example, referring to motor B, Fig. 4 shows the comparison between experimental measurements and the simulation results. Under low load condition the comparison confirms the satisfactory agreement between test results and predictions. Under high load condition, FEA overestimates the torque, probably due to a slightly different saturation effect of the iron. The offset on the average torque is lower than $4 \%$ and the waveforms resulting from FE simulation and measurements are showing the same oscillation. 


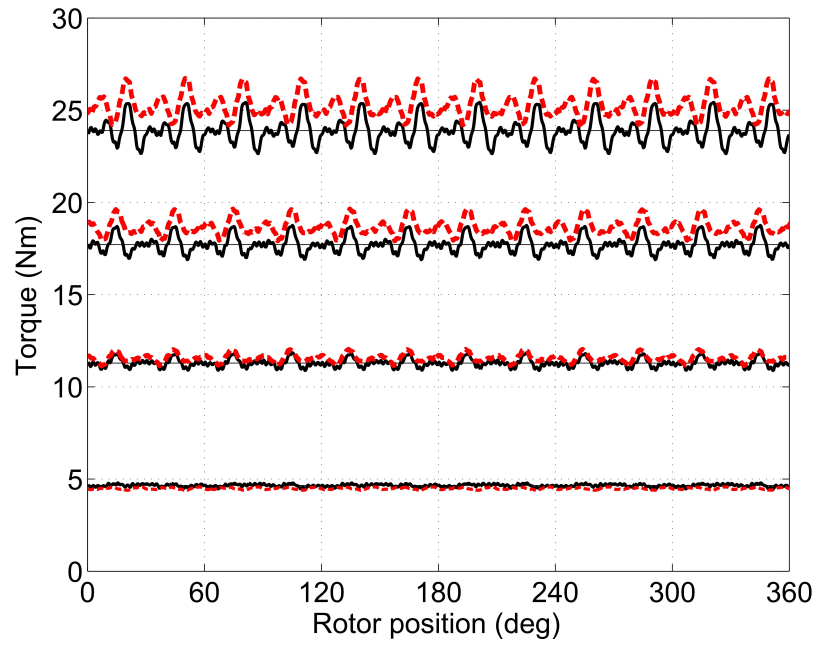

Fig. 4. Torque versus rotor position: experimental results (continuous line) vs FE simulation (dashed line), motor B).

\section{ANALYSIS OF TORQUE RIPPLE}

Torque behavior is calculated by means of finite element analysis, moving the rotor of 60 electrical degrees, corresponding to a torque ripple period for a three-phase machine. The stator windings are fed by given $I_{d}$ and $I_{q}$ currents. The electromagnetic torque is computed by means of the Maxwell stress tensor along the airgap surface. As previously mentioned, the main difficulty in designing a SynREL motor is to achieve an acceptable torque ripple. Torque ripple amplitude is defined as

$$
\Delta T=\frac{T_{\max }-T_{\min }}{T_{\text {avg }}}
$$

An example of torque vs. angular position for the motor A, is shown in Fig. 5. Fig. 5 also shows how the torque ripple varies according to the variation of flux barrier angles. A small variation from 37.5 to 39 degrees, of the third flux barrier angle $\vartheta_{b 3}$, has been considered.

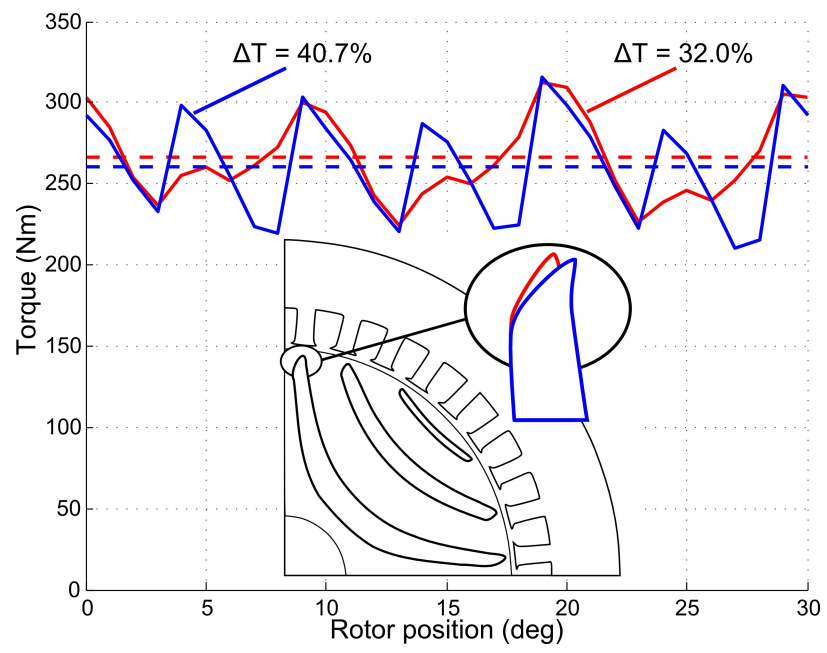

Fig. 5. Ripple torque due to a variation of the flux barrier angles in the airgap region (motor $\mathrm{A})$.
It can be noticed that while the average torque remains almost the same (the variation is less then $4 \%$ ), the variation of the third flux barrier angle can affect significantly the torque ripple ( $\Delta T$ varies from 32 to $40.7 \%$ ). To highlight the impact of such a variation it is useful to analyze the harmonic content of the torque ripple as shown in Fig. 6. In 36-slot 4-pole machine the higher torque ripple amplitudes are expected for harmonic of order 18, 36 etc which are the slot harmonics. The first configuration exhibits high torque harmonics corresponding to the order 18 and 36 (the slot harmonics). The second configuration exhibits a low torque harmonic of order 18, but a high harmonic of order 36 (amplitude of about $30 \mathrm{Nm}$ over an average torque of $260 \mathrm{Nm}$ ). The variation in the rotor fluxbarrier geometry yields an appreciable change in the torque harmonic distribution.
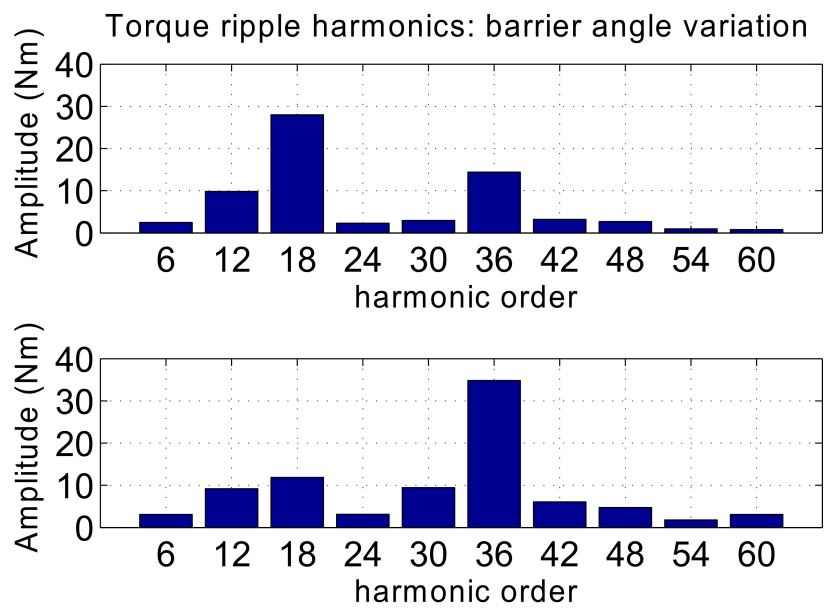

Fig. 6. Torque ripple harmonics comparison due to a variation of $\vartheta_{b 3}$ harmonic order refer to an electrical period, Motor A of Fig. 5

Similar analysis has been carried out on other motors. For instance let us refer to the motor C with $\vartheta_{b 1}=14.6 \mathrm{deg}, \vartheta_{b 2}$ $=26.3 \mathrm{deg}$ and $\vartheta_{b 3}=39 \mathrm{deg}$. A variation of two flux barrier ends is considered, with $\vartheta_{b 2}=26.1 \mathrm{deg}$ and $\vartheta_{b 3}=38.6 \mathrm{deg}$ respectively. As shown in Fig. 7, the amplitude of low order harmonics changes significantly.

Always referring to motor $\mathrm{C}$, another interesting comparison can be done for different airgaps, when the flux barrier ends angles are fixed $\left(\vartheta_{b 1}=14.6 \mathrm{deg}, \vartheta_{b 2}=26.3 \mathrm{deg}\right.$ and $\vartheta_{b 3}$ $=39 \mathrm{deg}$ ). The different harmonic contents are shown in Fig. 8. Increasing the airgap from 0.2 to $0.4 \mathrm{~mm}$, it is noticed the torque harmonic of 18th order growth and a smoothing effect for higher harmonics. A further effect of the airgap increase is the reduction (about 15\%) of the average torque and a consequent worsening of the relative torque ripple of about $5 \%$. These results highlight that small variations in rotor geometry cause substantial change in motor performance. For this reason, it is interesting to investigate the variation of the several design variables SynREL and PMAREL motors present. To this aim, it is useful to represent the variation of the torque ripple due to different combinations of design variables on a plane. 


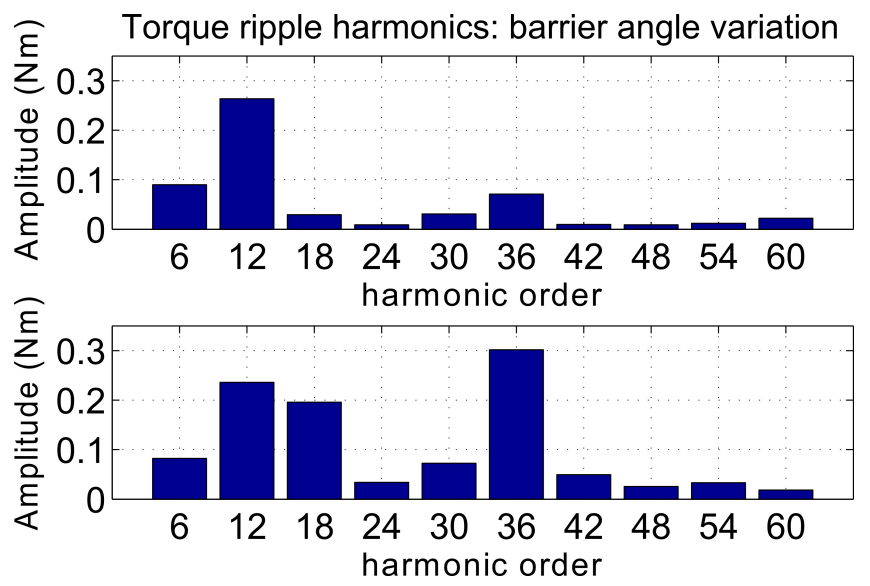

Fig. 7. Torque ripple harmonics comparison due to a variation of $\vartheta_{b 2}$ and $\vartheta_{b 3}$. Motor C, with symmetrical rotor.
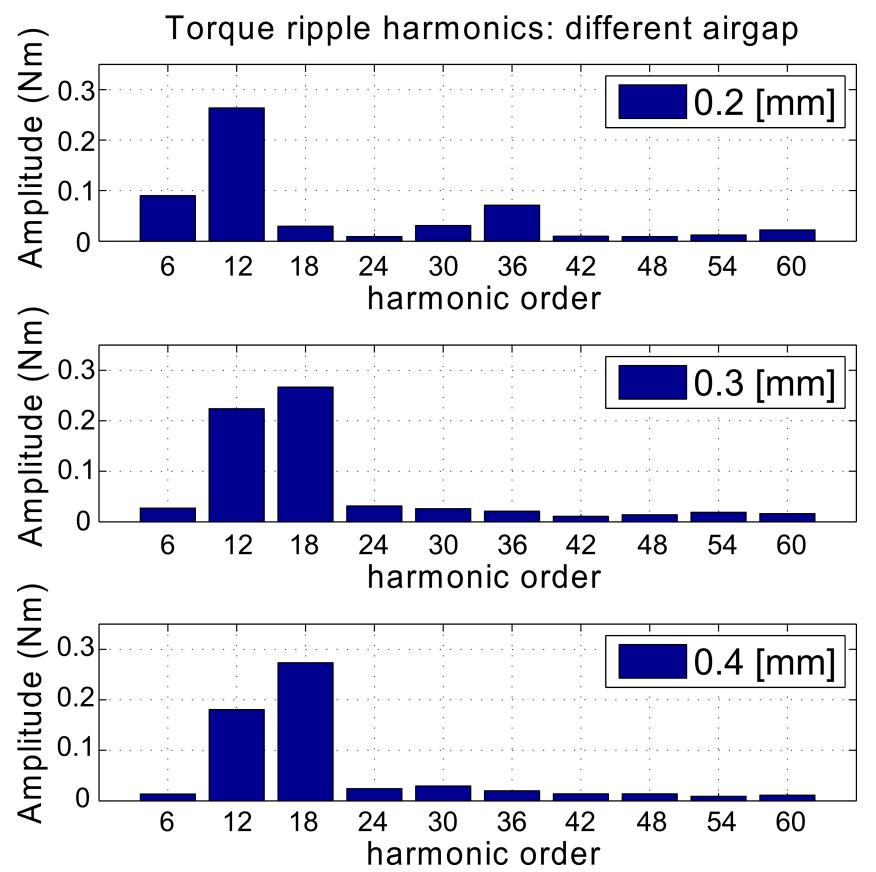

Fig. 8. Torque ripple harmonics comparison due to variation of the airgap Harmonic content of ripple torque referred to an electrical period: different airgap considered (Motor C).

At first, for a given flux barrier angle $\vartheta_{b 3}(38.8 \mathrm{deg})$ the impact of the flux barrier angles $\vartheta_{b 1}$ and $\vartheta_{b 2}$ on torque ripple is considered. The torque ripple resulting from $\mathrm{FE}$ analysis is represented on the variable plane shown in Fig. 9. In this case, the variation of $\vartheta_{b 1}$ and $\vartheta_{b 2}$ gives different effects on torque ripple. The variation of the first barrier angle $\vartheta_{b 1}$ does not change the torque ripple significantly. On the contrary, the variation of $\vartheta_{b 2}$ causes a variation up to $20 \%$ in the considered range. It is worth noticing that a 0.5 deg variation of $\vartheta_{b 2}$ (this is highlighted by black circles, from the optimal to the changed solution) leads to a variation of torque from 10 to $26 \%$.

Anyway, Fig. 10 shows that the resultant average torque seems to be not strongly affected by the flux barrier angles, remaining almost constant for each $\vartheta_{b 1}$ and $\vartheta_{b 2}$ combination.

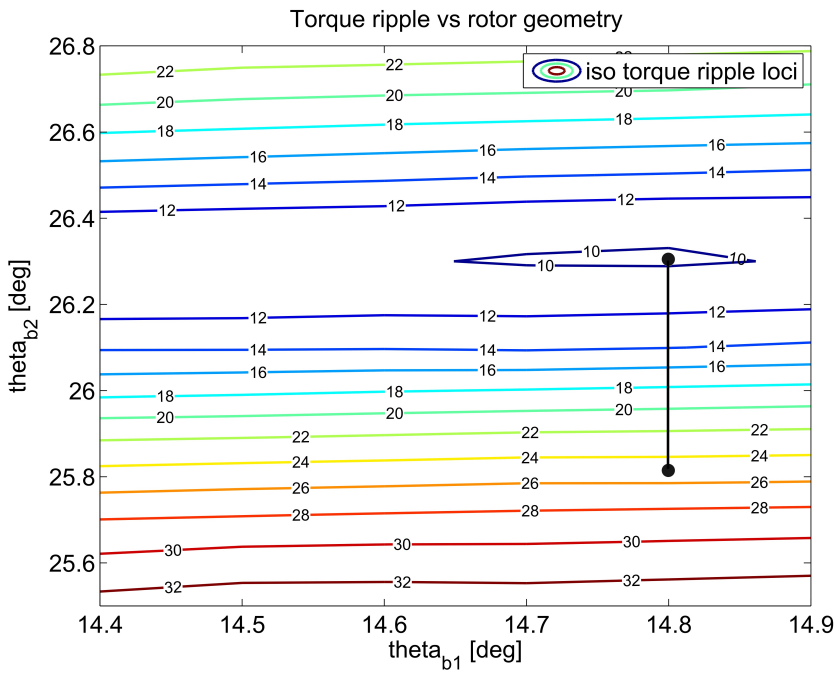

Fig. 9. Percentage torque ripple as a function of the design variables $\vartheta_{b 1}$ and $\vartheta_{b 2}$, with $\vartheta_{b 3}=38.8 \mathrm{deg}$ (Motor C).

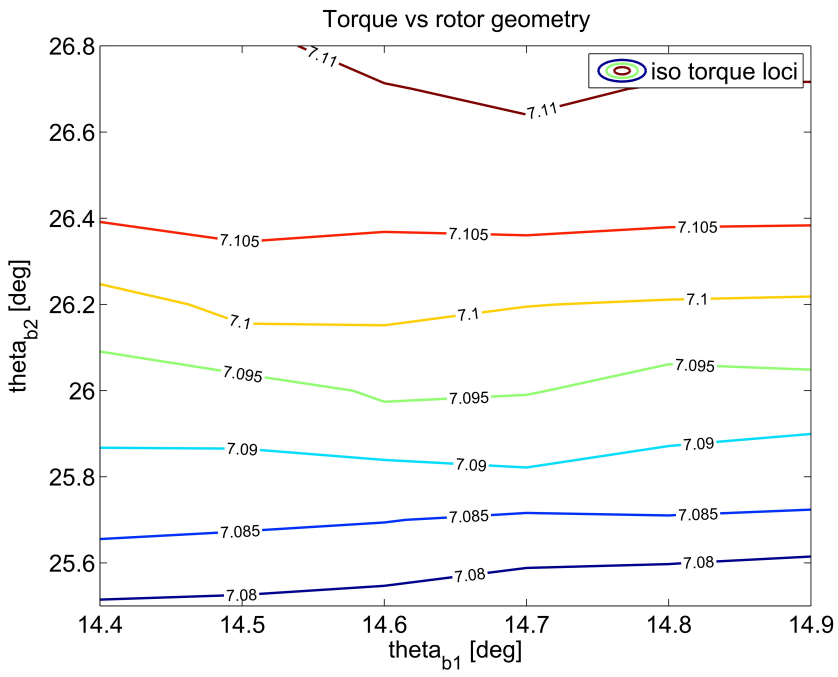

Fig. 10. Average torque as a function of the design variables $\vartheta_{b 1}$ and $\vartheta_{b 2}$, with $\vartheta_{b 3}=38.8 \mathrm{deg}$ (Motor C).

Similar results can be represented considering a variation of $\vartheta_{b 2}$ and $\vartheta_{b 3}$, while $\vartheta_{b 1}$ is fixed (14.8 deg), as represented in Fig. 11. The influence of the flux barrier angles on torque ripple depends on both $\vartheta_{b 2}$ and $\vartheta_{b 3}$. For some values of $\vartheta_{b 3}$, the variations of $\vartheta_{b 2}$ yield no effect on torque ripple, as shown in the bottom part of Fig. 11. For some other values of $\vartheta_{b 3}$, the angle $\vartheta_{b 2}$ has to be selected properly in order to minimize the torque ripple, as shown in the top part of Fig. 11. It is also worth noticing that a generic variation of $0.3 \mathrm{deg}$ for $\vartheta_{b 2}$ yields a higher torque ripple with respect to the same variation of $\vartheta_{b 3}$. Anyway, the average torque results to be not significantly affected by flux barrier angles variation.

It is worth noticing that, while other techniques used with the aim of reducing the torque ripple affect the average torque 
developed by the motor (e.g. shifting, shaping, skewing, stepskewing), the suitable choice of the flux barrier angles yields a reduction of the torque ripple only (as confirmed by the results obtained). The average torque remains fundamentally constant during the optimization process.

Combining the representation of all the flux barrier angles of Figs. 9 and 11, a set of variables giving an optimum solution can be carried out. Such a solution is matching the final result given by the optimization process (as discussed in Section IV). The results presented above are important in the first stage of the design process. They highlight those variables which have more influence on motor performance (i.e. $\vartheta_{b 2}$ and $\vartheta_{b 3}$ ) with respect to the others.

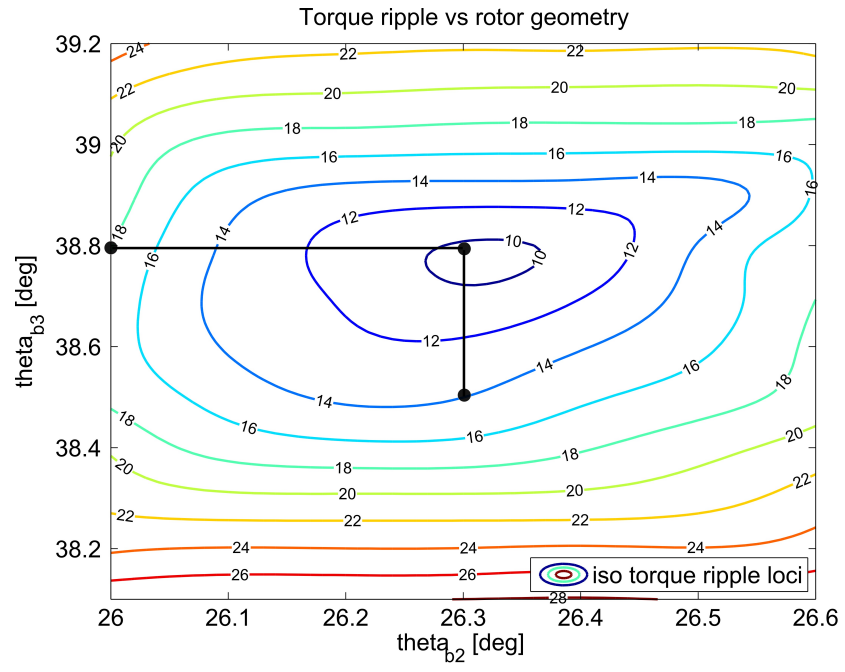

Fig. 11. Percentage torque ripple for different design variables: $\vartheta_{b 2}$ and $\vartheta_{b 3}$ with $\vartheta_{b 1}=14.8 \mathrm{deg}$ (Motor C)

Fig. 12 shows the percentage torque ripple as a function of the rotor outer diameter $D_{r e}$ (considering a fixed inner stator diameter) and the iron rib thickness. The larger $D_{r e}$ the smaller the airgap. Referring to the motor $\mathrm{C}$, a variation of $D_{r e}$ from 78.6 to $79.6 \mathrm{~mm}$, corresponds to an airgap variation between 0.75 and $0.2 \mathrm{~mm}$ respectively. For smaller airgap the influence of the iron ribs on the torque ripple is negligible. On the contrary the rib thickness exhibits a heavy influence on the torque ripple for large airgap values $\left(D_{r e}\right.$ lower than $78.9 \mathrm{~mm}$ ). Once again, for larger iron ribs, the torque ripple increases and the airgap could have some influence on it. It is also important to notice that there is an optimal airgap that minimizes the ripple (in this case $0.3-0.35 \mathrm{~mm}$ for motor C). It has been also noticed that the inner iron bridges instead have no effect on torque ripple. However, their thickness have to be minimized in order to avoid an average torque reduction and at the same time improve the power factor.

Fig. 13 shows the influence of the same variables on the average torque. The influence of the airgap is dominant with respect to the rib thickness. Similar results has been carried out for motor A.

The oscillation of the torque with respect to the average

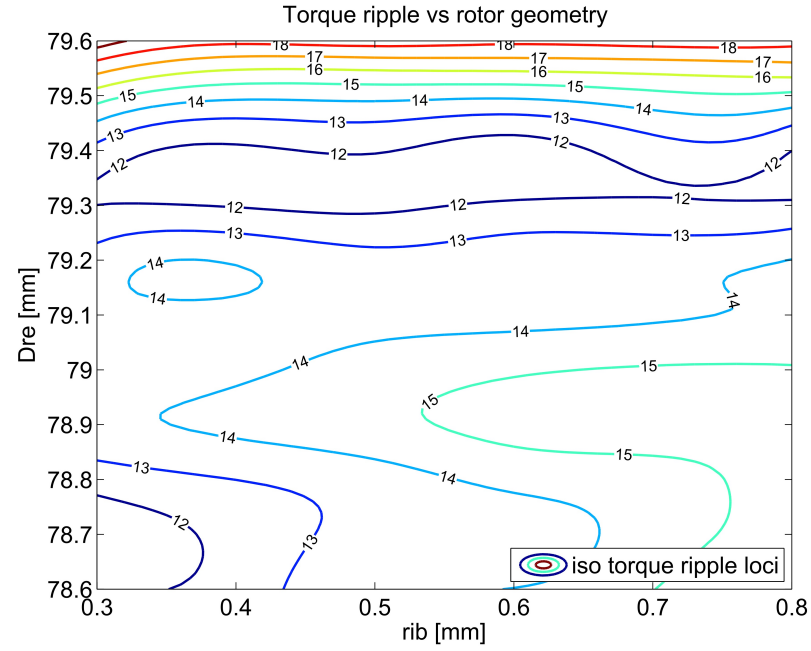

Fig. 12. Percentage torque ripple as a function of the airgap and the iron ribs thicknesses (motor $\mathrm{C}$ )

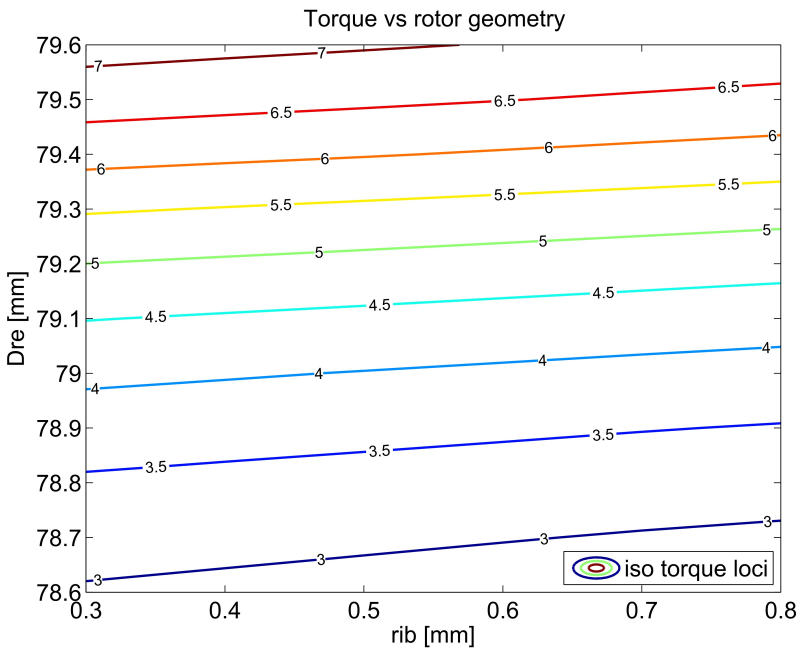

Fig. 13. Average torque as a function of the airgap and the iron ribs thicknesses (motor C).

value is also affected by other parameters related to the stator configuration at the air gap. For example, there is a negligible impact of the slot opening height $h_{s o}$. On the contrary, the larger the slot opening width $w_{s o}$ the greater the torque ripple.

\section{Optimized RELUCTANCE MACHINES UNDER ANALYSIS}

Once the main motor dimensions has been fixed, from the application constraints or by means of analytical design, an optimization of the motor is becoming a common practice. The optimization variables have to be selected. The choice of the variables, together with their number, is a key task to obtain a suitable final solution. Due to the high impact of both rotor and stator design parameters on the torque behavior, genetic algorithms (GA) optimizations have been carried out, considering the minimization of the torque ripple as objective function. 
TABLE II

OPTIMIZATION RESULTS (MOTOR C)

\begin{tabular}{cccc}
\hline Barrier angle & Initial & Final & unit \\
\hline$\vartheta_{b 1}$ & 12.5 & 14.8 & $\mathrm{deg}$ \\
$\vartheta_{b 2}$ & 27.5 & 26.3 & $\mathrm{deg}$ \\
$\vartheta_{b 3}$ & 37.5 & 38.8 & $\mathrm{deg}$ \\
& & & \\
\hline$\Delta T$ & 48.4 & 10.5 & $\%$
\end{tabular}

In particular, a strong impact of the angles of the flux barrier ends (i.e. $\vartheta_{b 1}, \vartheta_{b 2}, \vartheta_{b 3}$ ) has been found, as shown in the previous section. For this reasons, it makes sense to focus deeply on flux barrier geometry. As an example, Fig. 14 reports the torque behavior versus rotor position is reported for the initial geometry and for the final (optimized) solution. The main optimization input and output data are given in Table II.
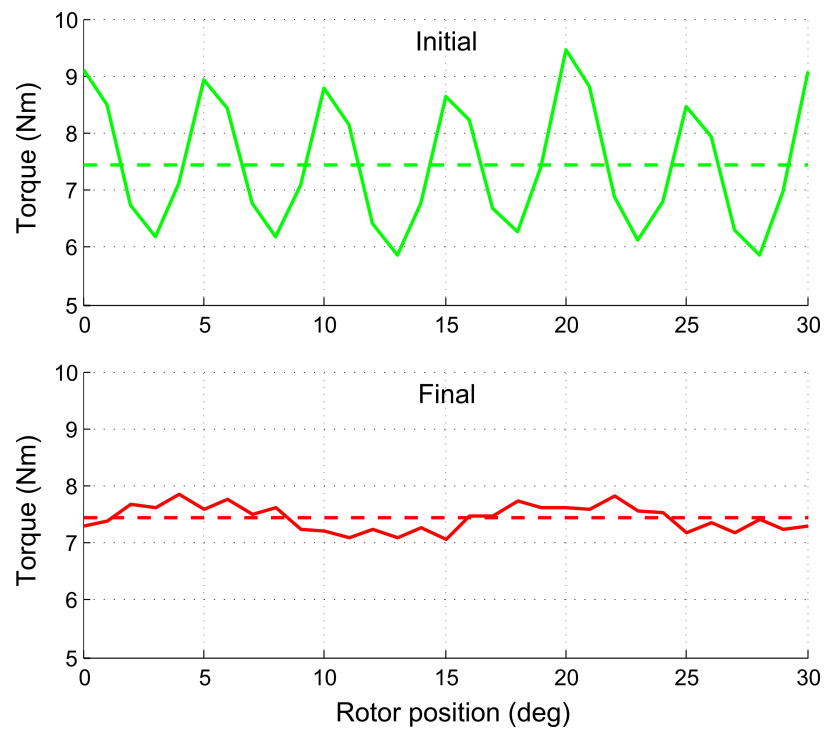

Fig. 14. Torque vs. rotor position behaviors before and after optimization (motor C): initial and final geometry.

Fig. 15 and Fig. 16 show the optimization results for the SynREL motors $A$ and $C$, with symmetrical rotor geometry. The angles of the flux barrier ends (the variables of the optimization) are plotted versus the corresponding torque ripple (the objective of the optimization). According to the objective of the torque ripple minimization, the best solutions are those on the left hand side of the figures. It is worth noticing that any variable converges to an optimal value in a tight range of variation. Thus, a set of best flux barrier angles can be done. The final optimization solutions for motors $\mathrm{A}$ and $\mathrm{C}$, gives a $\Delta T$ of about $16.5 \%$ and $10.5 \%$ respectively. The obtained ripples agree with typical values of relative torque ripple $\Delta T$ for SynREL and PMAREL machines (without rotor skewing). A skewing of the rotor geometry, applied to the optimized motor (with $\Delta T=10.5 \%$ ), led to a further reduction of torque oscillation, slightly reducing the average torque.
The same results are achieved for different motors, including the Machaon structure. In this case there are six flux barrier angles. The representation of Fig. 15 and Fig. 16 emphasizes that torque ripple increases (it almost doubles) with slight variations of flux barrier angles (they seem to be almost constant on the left hand side of the figures). In other words, slight variations of flux barrier angles cause a completely different behavior of the torque. This confirms the specific results shown in Figs. 5 to 8. These results highlight that once the optimal geometry is achieved, it is worth to evaluate the "robustness" of such a solution.

It is desirable that torque ripple results to be minimum even when occurring small variations of the working operating conditions (e.g., variation of current amplitude or phase) or small variations of the geometry (i.e. due to mechanical tolerance, wear of the machine tools, manufacturing or assembling inaccuracy, and so on). The sensitivity of the solutions found will be defined in Section V.

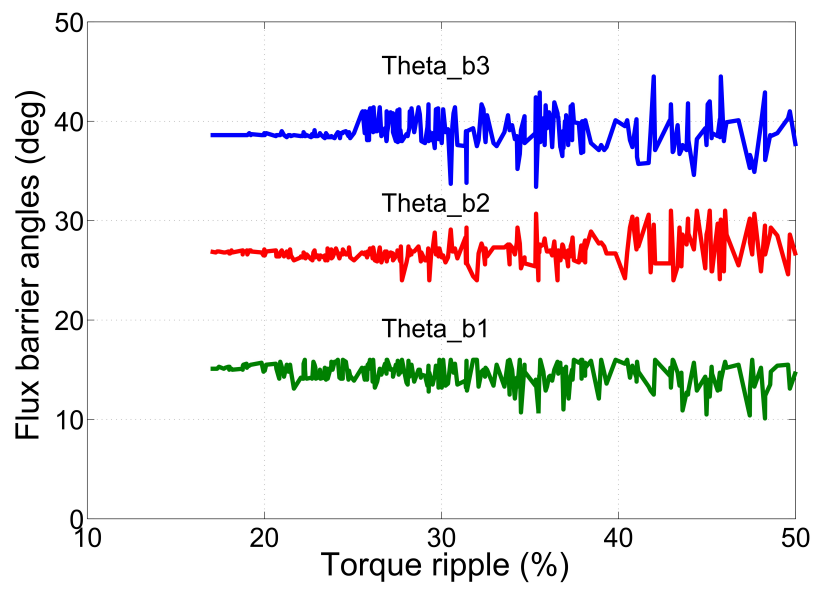

Fig. 15. Optimization direction of the flux barrier angles referring to the torque ripple (motor $\mathrm{A})$.

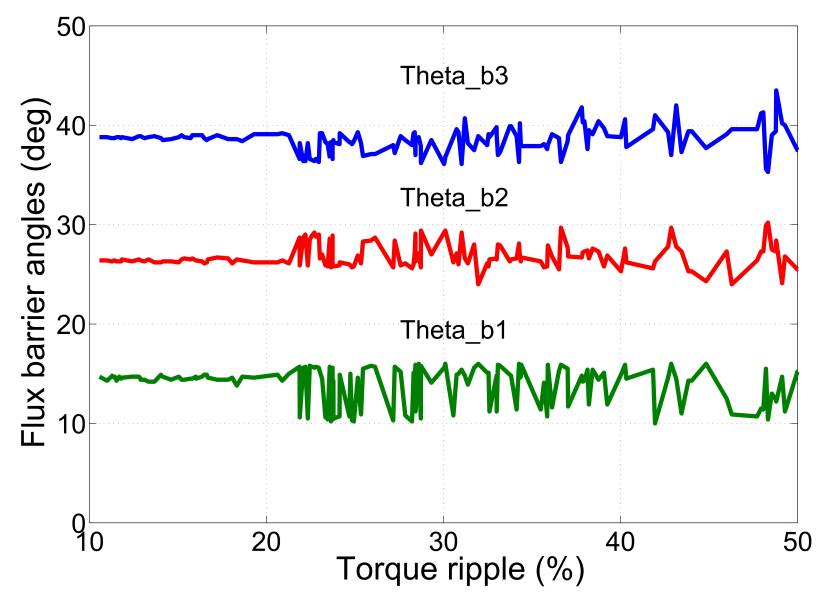

Fig. 16. Optimization direction of the flux barrier angles referring to the torque ripple (motor $\mathrm{C}$ ). 


\section{A. Torque ripple sensitivity}

At the end of the GA optimization, of both symmetric and Machaon configurations, several considerations about the torque ripple sensitivity to the parameters can be carried out. As a first step, the distance between two solutions is defined. A solution in the design space, e.g. the vector $\vec{x}$, is characterized by its $n_{v}$ variables (e.g., $x_{1}=\vartheta_{b 1}, x_{2}=\vartheta_{b 2}, x_{3}=\vartheta_{b 3}$ ). The distance between the vector $\vec{x}^{\prime}$ and the vector $\vec{x}^{\prime \prime}$ results in

$$
d\left(\vec{x}^{\prime}, \vec{x}^{\prime \prime}\right)=\sqrt{\sum_{i=1}^{n_{v}}\left({x_{i}^{\prime}}_{i}^{2}-x_{i}^{\prime \prime}\right)}
$$

Let us refer to the geometry which exhibits the minimum torque ripple, resulting from the GA minimization. It is defined by the vector $\overrightarrow{\tilde{x}}=\left(\tilde{x}_{1}, \tilde{x}_{2}, \ldots, \tilde{x}_{n}\right)$. The fluctuation of the torque ripple is computed according to the variation of the geometry with respect to the optimal solution $\overrightarrow{\tilde{x}}$, so as to evaluate the rate of change of the ripple with the deviation from the optimal solution, $\overrightarrow{\tilde{x}}$. Particularly important is the distance of a generic solution $\vec{x}$ from the optimal solution:

$$
d(\vec{x}, \overrightarrow{\tilde{x}})=\sqrt{\sum_{i=1}^{n_{v}}\left(x_{i}^{2}-\tilde{x}_{i}^{2}\right)}
$$

Figs. 17 and 18 show the torque ripple (1) versus the distance (3) from the optimal solution, which corresponds to the point characterized by geometry variation equal to zero.

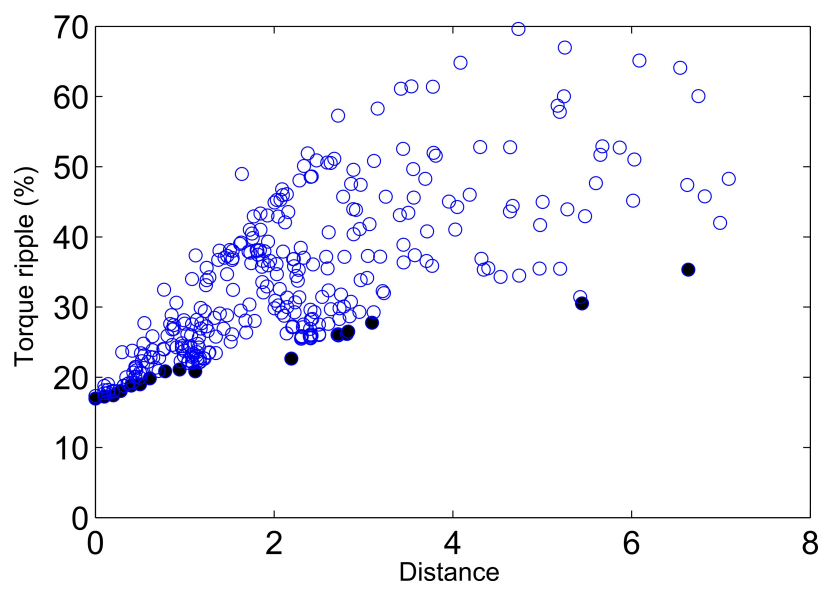

Fig. 17. Torque ripple vs distance for a symmetric configuration (motor A).

Fig. 17 refers to a symmetric rotor, Fig. 18 to a Machaon rotor. In the latter case, the distance is computed according to six flux barrier angles. The solutions obtained for the Machaon rotor gives a high number of solutions exhibiting low torque ripple as results comparing the black circles in Figs. 17 and 18. At distance zero, there is the optimal solution $\overrightarrow{\tilde{x}}$ and the torque ripple is the minimum ripple, found by means of the GA optimization. As the distance from the optimal solution increases, the torque ripple increases, too. Such behavior is evident for both configurations.

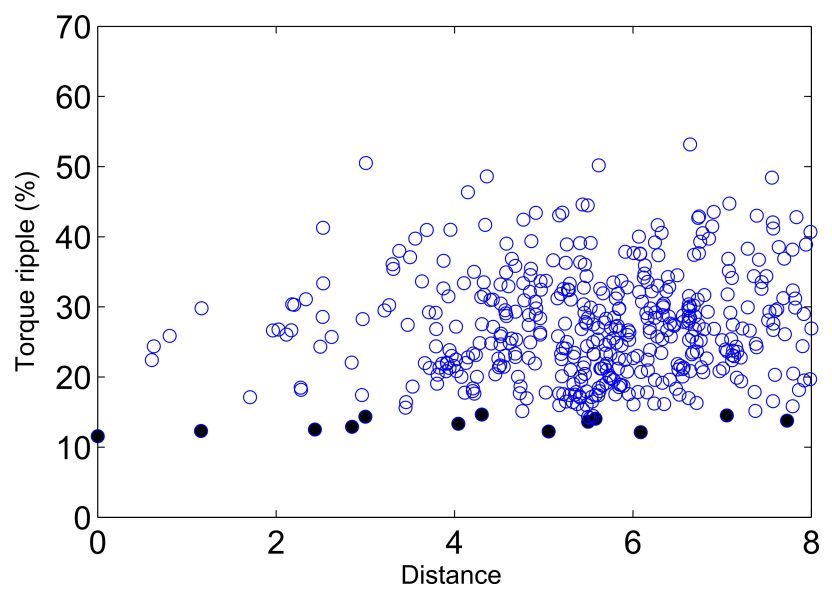

Fig. 18. Torque ripple vs distance for a Machaon configuration (motor C).

It is also worth noticing that the torque ripple increases rapidly even for small geometry variations. The upper limits of such representations correspond to the worst case solutions, i.e. the set of solutions exhibiting the highest sensitivity of torque ripple with respect to geometry variations. Among the two configurations, the rate of change is slightly lower with the Machaon rotor. The Machaon configuration reduces some torque harmonics that cause torque oscillation. Therefore, such a solution results to be slightly more robust from the sensitivity point of view.

\section{Sensitivity of The OPTIMAL SOlUtion}

In order to evaluate the impact of the variables on the machine performance of the SynREL motor, an evaluation of the sensitivity is presented, according to the criteria given in [20]. This is an inexpensive evaluation since it is based on the analysis of all the solutions found during the GA optimization process.

At first, a perturbation space is defined in the design variable space, based on the definition of a hypercube in $n_{v}$ dimensions.

The basic idea is to estimate the maximum variation rate of the torque ripple (the objective function) in a perturbation space (the $n_{v}$-dimension hypercube) centered in a given design vector $\vec{x}$ and composed of a number of feasible design vectors. For a given design vector $\vec{x}$, the associated hypercube is formed by all design vectors whose distance $d$ from $\vec{x}$ is lower than a fixed positive threshold.

Comparing all design points within the hypercube, the maximum and minimum value of the torque ripple is evaluated, i.e. $\Delta T_{\max }$ and $\Delta T_{\min }$. Then, the sensitivity of the torque ripple in the design point $\vec{x}$ is defined as their difference divided by the torque ripple achieved in the design point $\vec{x}$, center of the hypercube, that is, $\Delta T(\vec{x})$. It is

$$
s(\vec{x})=\frac{\Delta T_{\max }-\Delta T_{\min }}{\Delta T(\vec{x})}
$$

Therefore, such a sensitivity can be evaluated in all the design space, adopting the information from the solutions of 


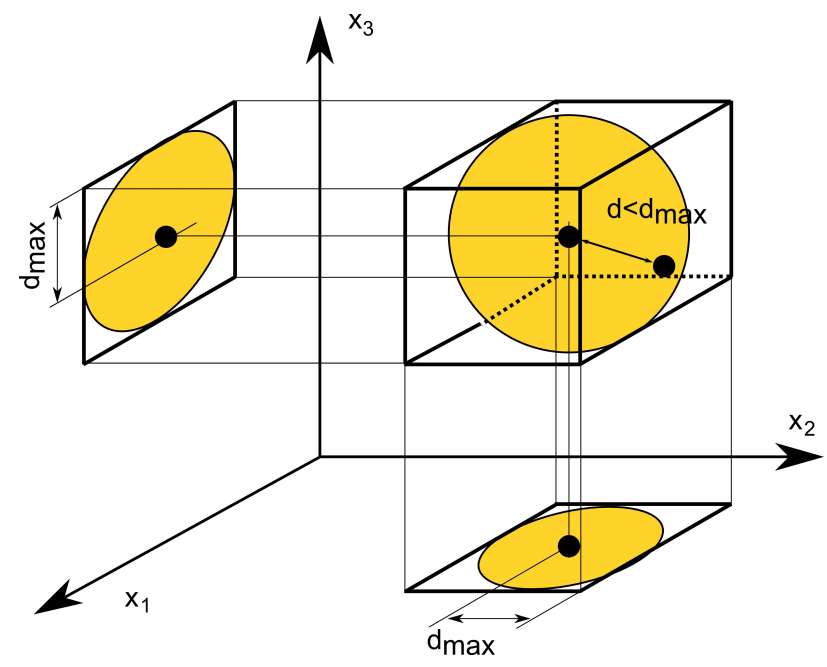

Fig. 19. Design vector centered in the hypercube space of the feasible solutions.

the GA optimizations that have been carried out. Fig. 20 shows the sensitivity defined in (4). The sensitivity to geometrical tolerances is higher and higher as the torque ripple is reduced. The lower the ripple torque, the higher the sensitivity. It becomes almost 10 times for the lower torque ripple. From the analysis, it seems that asymmetric rotor is slightly more robust than symmetric rotor, however the sensitivity to geometrical tolerances is high also in this case.

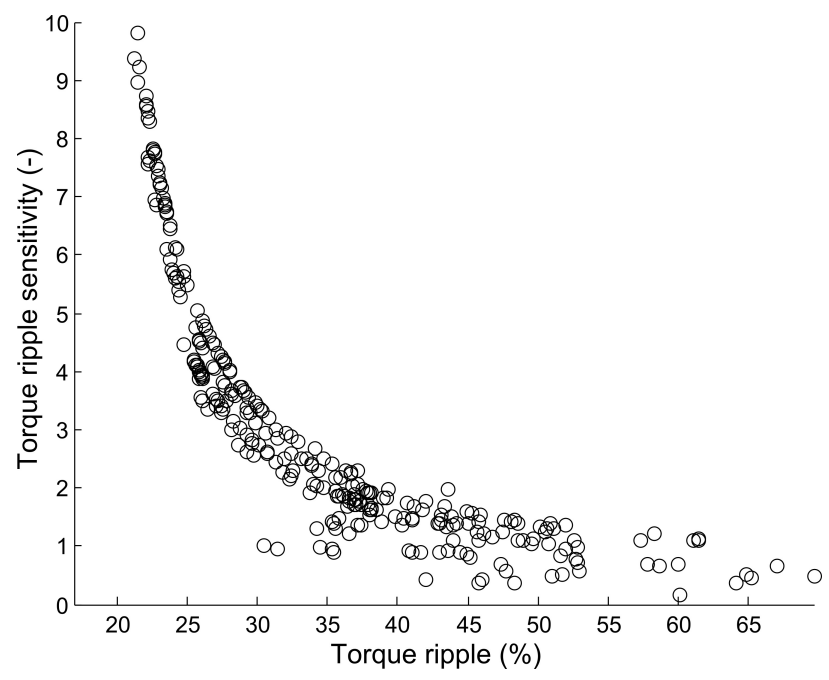

Fig. 20. Torque ripple sensitivity versus the percentage torque ripple.

\section{EFFECT OF THE PM ON TORQUE RIPPLE}

As said in the introduction PMs are commonly inset within the rotor flux barriers to saturate the iron bridges, improving the $\mathrm{PF}$ and the constant power speed range [21]. In this section the effect of the PM on the torque ripple is investigated. The PMAREL configurations considered, are based on the SynREL optimized motors (A and B) presented in section II-A. At first, the central part of the SynREL motors A and B has been filled with ferrite PMs as shown in Fig. 1. The map of the torque ripple behavior has been carried out increasing the PM dimensions by steps. Fig. 21 and Fig. 22 show the

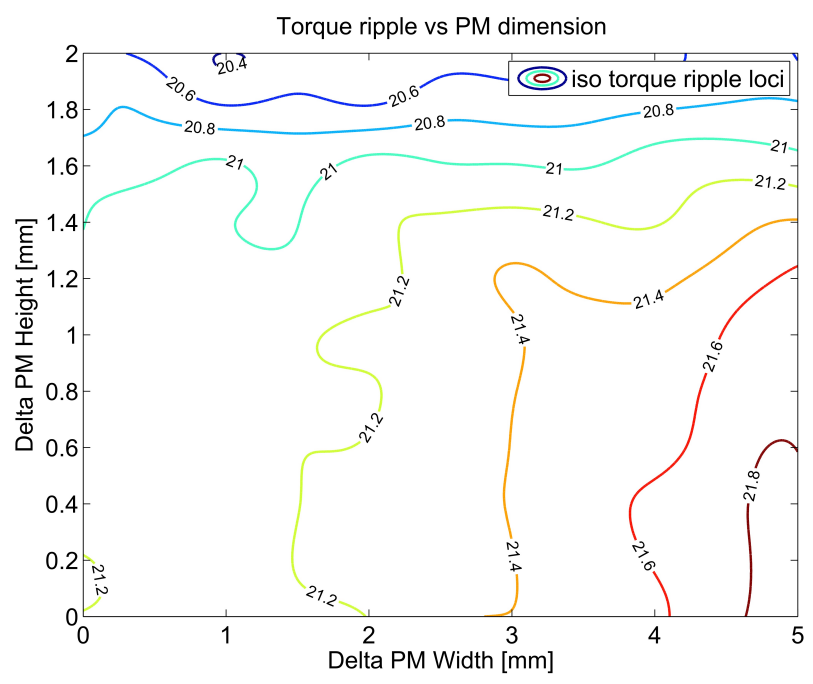

Fig. 21. PM dimensions effect on torque ripple (Motor A).

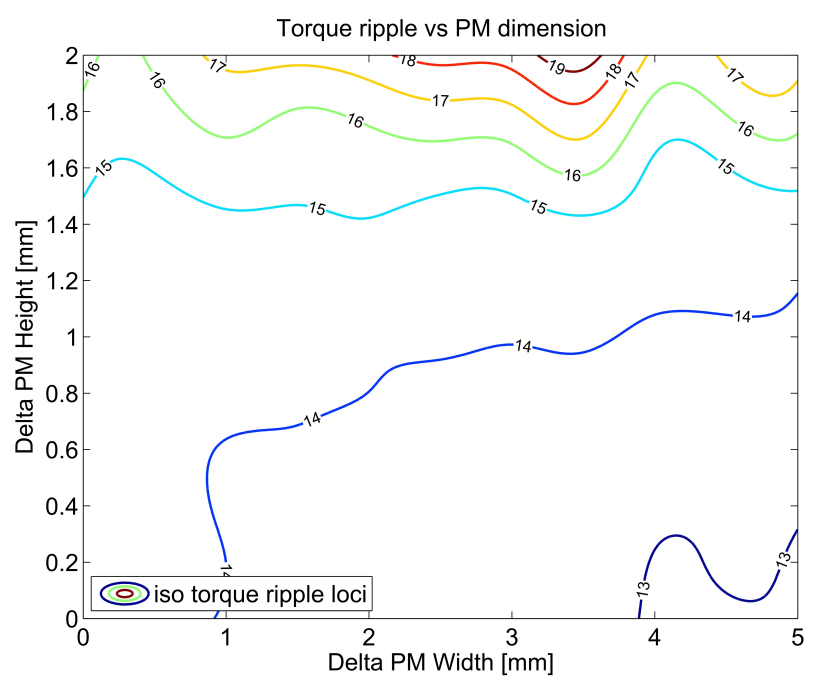

Fig. 22. PM dimensions effect on torque ripple (Motor B).

contour plots of the torque ripple, considering a variation of the PM height (vertical axis) and width (horizontal axis) referring to motor A and B respectively. It can be noticed that small variations of the PMs dimensions have a negligible impact on torque ripple. On the contrary, depending on the machine size, the impact of an increment of the PM height is higher. Referring to motor B (smaller than A), increasing the PM height of $2 \mathrm{~mm}$ yields a torque ripple variation from 13 to $18 \%$ (see Fig. 22).

\section{CONCLUSIONS}

This paper deals with the torque ripple reduction of SynREL and PMAREL machines. It is shown that torque ripple is 
strongly affected by stator and rotor geometry and by current phase and amplitude. Moreover, small geometry variations cause high torque ripple oscillations when "non-robust" solutions are adopted.

A detailed analysis of the design variables that have higher impact on the motor torque has been carried out showing their behavior for three different machines.

A novel method to evaluate the sensitivity of the torque ripple over all the design space has been presented, based on the analysis of the solutions resulting from the optimization process. The paper highlights the difficulties found in the design, even when an optimization procedure is carried out. It also provides some suggestions to be adopted in designing SynREL or PMAREL motors, to achieve more robust solutions as far as the torque ripple sensitivity is concerned. The analysis carried out for three different motor sizes shown the main parameters that have to be taken into account when designing a SynREL motor.

The effect of the PMs on the torque ripple has been investigated, showing a slightly greater impact of the PM height and a dependance on the machine size. In particular, the PMs dimension effects on torque ripple are significant for small motors. The measures on a prototype are in good agreement with the results predicted by means of finite element analysis.

\section{REFERENCES}

[1] A. Fratta, A. Vagati, and F. Villata. "Permanent magnet assisted synchronous reluctance drive for constant-power application: Drive power limit." In Proc. of Intelligent Motion European Conference, PCIM, pages 196-203, April Nurnberg, Germany, 1992.

[2] W.H. Kim, K.S. Kim, S.J. Kim, D.W. Kang, S.C. Go, Y.D. Chun, J. Lee, "Optimal PM Design of PMA-SynRM for Wide ConstantPower Operation and Torque Ripple Reduction," IEEE Transactions on Magnetics, vol. 45, no. 10, pp. 4660-4663, Oct. 2009.

[3] A. Fratta, GP. Troglia, A. Vagati, and F. Villata. "Evaluation of torque ripple in high performance synchronous reluctance machines." Records of IEEE Industry Application Society Annual Meeting, I:163170, October Toronto, Canada, 1993.

[4] A. Vagati, M. Pastorelli, G. Franceschini, and S.C. Petrache. "Design of low-torque-ripple synchronous reluctance motors." IEEE Trans. on Industry Application, IA-34(4):758-765, July-Aug. 1998.

[5] T.M. Jahns and W. L. Soong. "Pulsating torque minimization techniques for permanent magnet ac motor drives - a review." IEEE Trans. on Industrial Electronics, IE-43(2):321-330, April 1996.

[6] S.H. Han, T.M. Jahns, W.L. Soong, M.K. Guven, M.S. Illindala, "Torque Ripple Reduction in Interior Permanent Magnet Synchronous Machines Using Stators With Odd Number of Slots Per Pole Pair," IEEE Transactions on Energy Conversion, vol. 25, no. 1, pp.118-127, March 2010.

[7] N. Bianchi and S. Bolognani. "Reducing torque ripple in pm synchronous motors by pole shifting." Proceedings of International Conference on Electrical Machines, ICEM, Aug. Helsinki, 2000.
[8] M. Sanada, K. Hiramoto, S. Morimoto, and Y. Takeda, "Torque ripple improvement for synchronous reluctance motor using an asymmetric flux barrier arrangement," IEEE Transactions on Industry Applications, vol. 40, no. 4, pp. 1076-1082, Jul./Aug. 2004.

[9] T.Li and G.Slemon. "Reduction of cogging torque in permanent magnet motors." IEEE Trans. on Magnetics, 24(6):2901-2903, 1988

[10] N. Bianchi, S. Bolognani, D. Bon, and M. Dai Pré, "Rotor flux barrier design for torque ripple reduction in synchronous reluctance and pmassisted synchronous reluctance motors," IEEE Transactions on Industry Applications, vol. 45, no. 3, pp. 921 -928, may-june 2009.

[11] J.M. Park, S.I. Kim, J.P. Hong, J.H. Lee, "Rotor Design on Torque Ripple Reduction for a Synchronous Reluctance Motor With Concentrated Winding Using Response Surface Methodology," IEEE Transactions on Magnetics, vol. 42, no. 10, pp. 3479-3481, Oct. 2006

[12] F. Magnussen and H. Lendenmann, "Parasitic Effects in PM Machines With Concentrated Windings," IEEE Transactions on Industry Applications, vol. 43, no. 5, pp. 1223-1232, Sep./Oct. 2007.

[13] M. Barcaro and N. Bianchi, "Torque Ripple Reduction in FractionalSlot Interior PM Machines Optimizing the Flux-Barrier Geometries," in International Conference on Electrical Machines (ICEM), 2012, sept. 2012.

[14] D. Ionel. Interior permanent magnet motor including rotor with unequal poles. U.S. Patent, 8,102,091, Jan. 24, 2102.

[15] L. Alberti, M. Barcaro, and N. Bianchi "Design of a Low Torque Ripple Fractional-slot Interior Permanent Magnet Motor." in Conf. Rec. of the 2012 IEEE Energy Conversion Conference and Exposition, ECCE, Raleigh NC, USA, vol. 1, pp. 1-8, 2012.

[16] M. V. Cistelecan, F. J. T. E. Ferreira, and M. Popescu, "Three phase tooth-concentrated multiple-layer fractional windings with low space harmonic content," in 2010 IEEE Energy Conversion Congress and Exposition (ECCE), 2010, pp. $1399-1405$.

[17] L. Alberti and N. Bianchi, "Theory and design of fractional-slot multilayer windings," in Energy Conversion Congress and Exposition (ECCE), 2011 IEEE, sept. 2011, pp. $3112-3119$

[18] A. Vagati, B. Boazzo, P. Guglielmi, G. Pellegrino, "Design of FerriteAssisted Synchronous Reluctance Machines Robust Toward Demagnetization," in IEEE Transactions on Industry Applications, vol. 50, no. 3 , pp. 1768-1779, May-June 2014.

[19] Peng Zhang, D.M. Ionel, N.A.O. Demerdash, "Morphing Parametric Modeling and Design Optimization of Spoke and V-type Permanent Magnet Machines by Combined Design of Experiments and Differential Evolution Algorithms," in 2013 IEEE Energy Conversion Congress and Exposition, pp.5056-5063, 15-19, Sept. 2013.

[20] P. Di Barba, Multiobjective Shape Design in Electricity and Magnetism, Springer, Dordrecht, ISBN 978-90-481-3079-5, 2010.

[21] E. Carraro, M. Degano and N. Bianchi, "Permanent Magnet Volume Minimization in Permanent Magnet Assisted Synchronous Reluctance Motors," in International Conference on Ecological Vehicles and Renewable Energies (EVER), 2013, IEEE March 27-30, 2013, Monte-Carlo (Monaco).

[22] J. K. Tangudu and T. M. Jahns, "Comparison of Interior PM Machines with Concentrated and Distributed Stator Windings for Traction Applications," 2011 IEEE Vehicle Power and Propulsion Conf. (VPPC), pp. 1-8, 6-9 Sep. 2011.

[23] L. Chong and M. F. Rahman, "Saliency ratio derivation and optimisation for an interior permanent magnet machine with concentrated windings using finite-element-analysis," IET Electric Power Applications, vol. 4, no. 4, pp. 249-258, Apr. 2010. 